Article

\title{
Construction of Self-Healing Disulfide-Linked Silicone Elastomers by Thiol Oxidation Coupling Reaction
}

\author{
Yanhua Huang ${ }^{1}$, Jianpan Yan ${ }^{2}$, Dengxu Wang ${ }^{2, * \mathbb{C}}$, Shengyu Feng ${ }^{2}$ and Chuanjian Zhou ${ }^{1,2, *}$ \\ 1 School of Materials Science and Engineering, Shandong University, Jinan 250022, China; hyhsdu@163.com \\ 2 National Engineering Research Center for Colloidal Materials, Key Laboratory of Special Functional \\ Aggregated Materials, Ministry of Education, Shandong Key Laboratory of Advanced Organosilicon \\ Materials and Technologies, School of Chemistry and Chemical Engineering, Shandong University, \\ Jinan 250100, China; jpanyan@163.com (J.Y.); fsy@sdu.edu.cn (S.F.) \\ * Correspondence: dxwang@sdu.edu.cn (D.W.); zhouchuanjian@sdu.edu.cn (C.Z.)
}

check for updates

Citation: Huang, Y.; Yan, J.; Wang, D.; Feng, S.; Zhou, C. Construction of Self-Healing Disulfide-Linked Silicone Elastomers by Thiol Oxidation Coupling Reaction. Polymers 2021, 13, 3729. https:// doi.org/10.3390/polym13213729

Academic Editor: Andrea Sorrentino

Received: 30 September 2021

Accepted: 26 October 2021

Published: 28 October 2021

Publisher's Note: MDPI stays neutral with regard to jurisdictional claims in published maps and institutional affiliations.

Copyright: (c) 2021 by the authors. Licensee MDPI, Basel, Switzerland. This article is an open access article distributed under the terms and conditions of the Creative Commons Attribution (CC BY) license (https:// creativecommons.org/licenses/by/ $4.0 /)$.

\begin{abstract}
Developing self-healing silicone elastomers are highly significant because of their promising applications. Herein, we present novel self-healing disulfide-linked silicone elastomers (SEs) based on thiol-terminated sulfur-containing heterochain polysiloxanes (P-SHs) and three thiolcontaining crosslinkers, including pentaerythritol tetrakis( $\beta$-mercaptopropionate) (PETMP), octa (3-mercaptopropyl)silsesquioxane (POSS-SH), and poly[(mercaptopropyl)methylsiloxane] (PMMS), via the thiol oxidation coupling reactions. The construction of these SEs can rapidly proceed at room temperature. The effects of crosslinker species and amounts on the formability and mechanical properties were investigated. The silicone elastomers can be self-healed by heating at $150{ }^{\circ} \mathrm{C}$ for $2 \mathrm{~h}$ or under UV radiation for $30 \mathrm{~min}$ after cutting them into pieces and the self-healing efficiency is $>70 \%$. Moreover, they can be utilized as adhesives for bonding glass sheets, which can hold a $200 \mathrm{~g}$ weight. The bonding is reversible and can repeatedly proceed many times, indicating that these materials can promisingly be applied as reversible adhesives. These results indicate that a thiol oxidation coupling reaction is a simple and effective strategy for the construction of self-healing disulfide-linked elastomers. Under this strategy, more disulfide-linked organic elastomers with self-healing properties can be designed and constructed and their applications can be further explored.
\end{abstract}

Keywords: silicone elastomers; self-healing; thiol oxidation coupling reaction; disulfide bond; adhesives

\section{Introduction}

Silicone elastomers (SEs) as an important class of organosilicon materials have attracted significant interest due to their intriguing properties, such as high/low temperature resistance, high surface activity, electric resistance, and biocompatibility, and have found extensive applications in aerospace, electronics, coatings, adhesives, medicine, and so forth [1-4]. Recently, SEs with a self-healing property have gained particular attention because this property not only can lead to the extension of their usable life, recycling, and reduction of waste, but also can meet their high requirements in emerging applications, such as stretchable electronics, wearable strain sensors, and soft robots [5-7]. The SEs with a self-healing property are typically realized by pre-embedded a healing agent in the system (called extrinsic self-healing), or incorporating dynamic linkers in the networks (called intrinsic self-healing) [6]. In recent years, most of the efforts have been devoted to develop the intrinsic self-healing SEs because the diversified dynamic interactions, including reversible covalent bonds (such as disulfide [8], boroxine [9], imine [10], a Diels-Alder reaction [11], transesterification [12]), coordination bonds [13], hydrogen bonds [14], $\pi-\pi$ stacking interactions [15], and host-guest interactions [16]) can be readily incorporated in the networks and the obtained SEs can be repeatedly healed. 
Among various dynamic interactions, disulfide bonds-as a typical reversible bond-have been widely applied to construct self-healing materials as their reversible nature can be easily triggered by various conditions, such as heating [17,18], UV light [19], sunlight [20,21], and alkaline [22]. In addition, by virtue of disulfide bonds, novel selfhealing SEs were also achieved [23-25]. For example, Zhang et al. reported sulfide-linked SEs using $\alpha, \omega$-dihydroxyl polydimethylsiloxane as the base polymer and commercially available bis(triethoxysilylpropyl) disulfide as the crosslinker [23]. The resultant SEs can be self-healed and recycled multiple times without any catalyst under sunshine. By introducing aromatic disulfides into a siloxane matrix, a stretchable, fast-healing SE exhibiting a tensile stress of $0.5 \mathrm{MPa}$ and a healing efficiency above $95 \%$ at room temperature has been constructed [26]. Nevertheless, the disulfide bonds in these SEs were commonly formed before the crosslinking. In other words, disulfide-containing monomers were commonly utilized as the crosslinkers for the SEs. There is still no report on self-healing SEs crosslinked by a disulfide bond, which is directly formed during the crosslinking.

Herein, we introduce a thiol oxidation coupling reaction as the crosslinking strategy to construct disulfide bond crosslinked SEs using thiol-terminated sulfur-containing heterochain polysiloxane as the base polymer and thiol-containing compounds or polymers as the crosslinkers. The mechanical properties of resultant SEs can be tuned by altering the amount and species of crosslinkers. By taking advantages of reversible disulfide bonds in the networks, the SEs can be self-healed under heating or UV irradiation. In addition, their application as adhesives was also explored.

\section{Materials and Methods}

\subsection{Materials and Characterization}

We purchased 1,3-divinyl-1,1',3,3'-tetramethyldisiloxane (99\%), 1,2-ethanedithiol (99\%), and 2,2-dimethoxy-2-phenylacetophenone (98\%), and pentaerythritol tetrakis( $\beta$-mercaptopropionate) (95\%) from Energy Chemical (Shanghai, China). Sodium iodide (99\%) and 30\% $\mathrm{H}_{2} \mathrm{O}_{2}$ aqueous solution were purchased from Tianjin Kemiou Chemical Reagent Co., Ltd. (Tianjin, China) and Laiyang Fine Chemicals (Laiyang, China), respectively. The commercially available reagents were used without further purification. Poly[(mercaptopropyl) methyldimethylsiloxane] (PMMS) was prepared as in our previous report [27]. Octa (3-mercaptopropyl)silsesquioxane (POSS-SH) was synthesized as a previous report and the purity is $>99 \%$ based on the elemental analysis [28]. Fourier transform infrared (FTIR) spectra were measured within a 4000 to $400 \mathrm{~cm}^{-1}$ region on a Bruker TENSOR-27 infrared spectrophotometer (KBr pellet) (Ettlingen, Germany). ${ }^{1} \mathrm{H}$ NMR were measured on a Bruker AVANCE-400 NMR spectrometer (Rheinstetten, Germany) using $\mathrm{CDCl}_{3}$ as the solvent and without tetramethylsilane (TMS) as an internal reference. Elemental analyses were conducted using an Elementar vario EL III elemental analyzer (Munich, Germany). Thermogravimetric analysis (TGA) (Mettler Toledo, Columbus, OH, USA) was performed under $\mathrm{N}_{2}$ using a TA SDTQ600 at a temperature range of room temperature to $600{ }^{\circ} \mathrm{C}$ with a heating rate of $10^{\circ} \mathrm{C} \mathrm{min}^{-1}$. The differential scanning calorimetry (DSC) (Mettler Toledo, Columbus, $\mathrm{OH}, \mathrm{USA}$ ) was conducted under $\mathrm{N}_{2}$ using a Netzsch DSC 204 Phoenix at a temperature range of -100 to $150{ }^{\circ} \mathrm{C}$ with a heating rate of $10^{\circ} \mathrm{C} \cdot \mathrm{min}^{-1}$. The tensile properties of elastomers were tested using the 3340 model Instron universal testing machine at room temperature with a test speed of $100 \mathrm{~mm} / \mathrm{min}$.

\subsection{Synthesis of Thiol-Terminated Sulfur-Heterochain Polysiloxane (P-SH-1 to P-SH-4)}

P-SH-1: 1,3-divinyl-1,1',3,3'-tetramethyldisiloxane (MM $\left.{ }^{\mathrm{Vi}}\right)(18.64 \mathrm{~g}, 0.1 \mathrm{~mol})$, 1,2-ethanedithiol (EDT) (11.30 g, $0.12 \mathrm{~mol}$ ), and 2,2-dimethoxy-2-phenylacetophenone (DMPA) (0.3 g) were added to a $50 \mathrm{~mL}$ round-bottom flask. The mixture was stirred for $30 \mathrm{~min}$ while being exposed to UV light at a wavelength of $365 \mathrm{~nm}$. The viscosity of the mixture gradually increased. Then cold methanol was added to the mixture to wash the unreacted reagents and DMPA three times. After removing the methanol under vacuum, the product was obtained as a colorless and viscous liquid (yield: $91.5 \%, 27.40 \mathrm{~g}$ ). 
P-SH-2: the synthesis and post-treatment of P-SH-2 were similar to those of P-SH-1 except that the amount of EDT was $10.36 \mathrm{~g}(0.11 \mathrm{~mol})$ with a molar ratio of EDT and MM $\mathrm{Mi}^{\mathrm{Vi}}$ as 1.1:1. The product was obtained as a colorless and viscous liquid (yield: $92.5 \%, 26.82 \mathrm{~g}$ ).

P-SH-3: the synthesis and post-treatment of P-SH-3 were similar to those of P-SH-1 except that the amount of EDT was $9.89 \mathrm{~g}(0.105 \mathrm{~mol})$ with a molar ratio of EDT and MM $\mathrm{Mi}^{\mathrm{Vi}}$ as 1.05:1. The product was obtained as a colorless and viscous liquid (yield: $92.4 \%, 26.36 \mathrm{~g}$ ).

P-SH-4: the synthesis and post-treatment of P-SH-4 were similar to those of P-SH-1 except that the amount of EDT was $9.51 \mathrm{~g}(0.101 \mathrm{~mol})$ with the molar ratio of EDT and $\mathrm{MM}^{\mathrm{Vi}}$ as 1.01:1. The product was obtained as a colorless and viscous liquid (yield: $92.4 \%$, $26.36 \mathrm{~g})$.

${ }^{1} \mathrm{H}$ NMR $\left(400 \mathrm{MHz}, \mathrm{CDCl}_{3}\right): \delta 2.63-2.76\left(\mathrm{~m},-\mathrm{SCH}_{2} \mathrm{CH}_{2} \mathrm{SH}\right), 2.52-2.60(\mathrm{~m}$, $\left.-\mathrm{SiCH}_{2} \mathrm{CH}_{2} \mathrm{~S}-\right)$, 1.24-1.29 (m, - SH),0.82-0.90 (m, $\left.-\mathrm{SiCH}_{2} \mathrm{CH}_{2} \mathrm{~S}-\right), 0.01-0.14\left(\mathrm{~m},-\mathrm{SiCH}_{3}\right)$.

The molecular weights of P-SH-1 to P-SH-4 were 1500, 2900, 5700, and 28,100 g/mol, which were calculated based on the results of ${ }^{1} \mathrm{H}$ NMR.

\subsection{General Synthesis of Disulfide-Linked SES}

In a typical synthesis, P-SH-1 (2 g, $1.34 \mathrm{mmol})$, pentaerythritol tetrakis( $\beta$-mercaptopropionate) (PETMP) $(65.4 \mathrm{mg}, 0.134 \mathrm{mmol})$, and tetrahydrofuran (THF) $(10 \mathrm{~mL})$ were placed into a weighing bottle and mixed under stirring. Then $\mathrm{NaI}$ in aqueous solution $(0.05 \mathrm{~mL}$, $0.03 \mathrm{mmol}, 0.1 \mathrm{~g} / \mathrm{mL}$ ) and $30 \% \mathrm{H}_{2} \mathrm{O}_{2}$ aqueous solution $(0.1 \mathrm{~mL})$ were added in the solution and stirred for $50 \mathrm{~min}$ at room temperature. The resultant mixture was poured into a Teflon mold and remained at room temperature. The resultant crude elastomer was washed by $5 \%$ sodium thiosulfate aqueous solution three times to remove the residual catalyst and oxidant. Finally, the product was dried at $90{ }^{\circ} \mathrm{C}$ in a vacuum oven for $24 \mathrm{~h}$ and afforded as a transparent elastomer (SE-4). Warning: the $30 \% \mathrm{H}_{2} \mathrm{O}_{2}$ aqueous solution should be carefully taken and used because it can potentially cause fires and explosions!

Other SEs were achieved under a similar procedure of SE-4 except for the formulation of the starting materials. Table 1 summarizes the variables that were examined to study the correlation of formability of SEs with the reagents. The factors include the types of P-SHs and crosslinkers, and the amounts of crosslinkers.

Table 1. Experimental data on the preparation of silicone elastomers under different conditions.

\begin{tabular}{|c|c|c|c|c|c|c|}
\hline Entry & P-SHs ${ }^{a}$ & $\mathrm{THF} / \mathrm{mL}$ & $\mathrm{H}_{2} \mathrm{O}_{2 /} \mathrm{mL}^{b}$ & $\mathrm{NaI} \mathrm{mL}{ }^{c}$ & Crosslinkers (g) & Formability \\
\hline SE-1 & P-SH-1 & 8 & 0.1 & 0.05 & PETMP(0) & $\times($ tacky $)$ \\
\hline SE-2 & P-SH-1 & 8 & 0.1 & 0.05 & $\operatorname{PETMP}(0.016)$ & $\times($ tacky $)$ \\
\hline SE-3 & P-SH-1 & 8 & 0.1 & 0.05 & PETMP(0.036) & $\sqrt{ }$ \\
\hline SE-4 & P-SH-1 & 8 & 0.1 & 0.05 & PETMP(0.065) & $\sqrt{ }$ \\
\hline SE-5 & P-SH-1 & 8 & 0.1 & 0.05 & $\operatorname{PETMP}(0.1)$ & $\sqrt{ }($ cracks inside $)$ \\
\hline SE-6 & P-SH-1 & 8 & 0.1 & 0.05 & POSS-SH(0.01) & $\sqrt{ }$ \\
\hline SE-7 & P-SH-1 & 8 & 0.1 & 0.05 & POSS-SH(0.02) & $\sqrt{ }$ \\
\hline SE-8 & P-SH-1 & 8 & 0.1 & 0.05 & POSS-SH(0.04) & $\sqrt{ }$ \\
\hline SE-9 & P-SH-1 & 8 & 0.1 & 0.05 & POSS-SH(0.06) & $\sqrt{ }$ \\
\hline SE-10 & P-SH-1 & 8 & 0.1 & 0.05 & $\operatorname{PMMS}(0.10)$ & $x$ \\
\hline SE-11 & P-SH-2 & 8 & 0.1 & 0.05 & PETMP(0.0168) & $\times($ tacky, bubbles inside $)$ \\
\hline SE-12 & P-SH-2 & 8 & 0.1 & 0.05 & $\operatorname{PETMP}(0.036)$ & $\sqrt{ }$ \\
\hline SE-13 & P-SH-2 & 8 & 0.1 & 0.05 & PETMP(0.068) & $\sqrt{ }$ \\
\hline SE-14 & P-SH-2 & 8 & 0.1 & 0.05 & POSS-SH(0.06) & $\sqrt{ }$ \\
\hline SE-15 & P-SH-3 & 8 & 0.1 & 0.05 & PETMP(0.0168) & $\times($ tacky, bubbles inside $)$ \\
\hline SE-16 & P-SH-3 & 8 & 0.1 & 0.05 & PETMP(0.036) & $\sqrt{ }$ (bubbles inside) \\
\hline SE-17 & P-SH-3 & 8 & 0.05 & 0.05 & PETMP(0.036) & $\sqrt{ }$ \\
\hline SE-18 & P-SH-3 & 8 & 0.05 & 0.05 & PETMP(0.068) & $\sqrt{ }$ (bubbles inside) \\
\hline SE-19 & P-SH-3 & 8 & 0.05 & 0.05 & POSS-SH(0.02) & $\times($ tacky, bubbles inside $)$ \\
\hline SE-20 & P-SH-3 & 8 & 0.05 & 0.05 & POSS-SH(0.04) & $\sqrt{ }$ \\
\hline SE-21 & P-SH-3 & 8 & 0.05 & 0.05 & POSS-SH(0.06) & $\sqrt{ }($ cracks inside $)$ \\
\hline SE-22 & P-SH-4 & 8 & 0.1 & 0.05 & PETMP/POSS-SH & $\times($ tacky $)$ \\
\hline
\end{tabular}

${ }^{a}$ All the amounts of P-SHs used in the formulations are $2 \mathrm{~g} ;{ }^{b}$ the concentration of $\mathrm{H}_{2} \mathrm{O}_{2}$ in aqueous solution is $30 \%$; ${ }^{c}$ the concentration of $\mathrm{NaI}$ in aqueous solution is $0.1 \mathrm{~g} / \mathrm{mL}$. " $\times$ " and " $\sqrt{ }$ " mean that the elastomers cannot be formed and be formable. 


\subsection{Lap Shear Adhesion}

The lap shear adhesion referred to a previous report with some modification [18]. Lap joints were made by placing a $6 \mathrm{~mm} \times 6 \mathrm{~mm}$ square of elastomer between two overlapping $25 \mathrm{~mm} \times 75 \mathrm{~mm}$ glass sheets. The resultant sheets were clamped using two clamps and exposed to UV light for $30 \mathrm{~min}$. During this adhesive process, contact pressure was maintained. After removing the UV irradiation, the adhesive glass sheets were applied to hold different weights.

\section{Results and Discussion}

\subsection{Synthesis and Characterization}

As shown in Scheme 1, the thiol-terminated sulfur-containing heterochain polysiloxane (P-SHs) were firstly synthesized by the thiol-ene reaction of 1,3-divinyl-1, $1^{\prime}, 3,3^{\prime}$ tetramethyldisiloxane $\left(\mathrm{MM}^{\mathrm{Vi}}\right)$ and 1,2-ethanedithiol (EDT) with DMPA as the initiator under UV-light. To control the molecular weights of P-SHs, the molar ratios of EDT and $\mathrm{MM}^{\mathrm{Vi}}$ were altered from 1.2:1 to 1.01:1. Then the sulfide-linked SEs were synthesized by the thiol oxidation coupling reactions, while using P-SHs as the base polymers and three thiol-containing compounds or polymers, including PETMP, POSS-SH, and PMMS, as the crosslinkers. It was found that the crosslinking process can rapidly occur at room temperature. After removing the unreacted reagents and catalyst, the final products were afforded and the formability of these elastomers depends on the formulations (Table 1, see the following discussion).

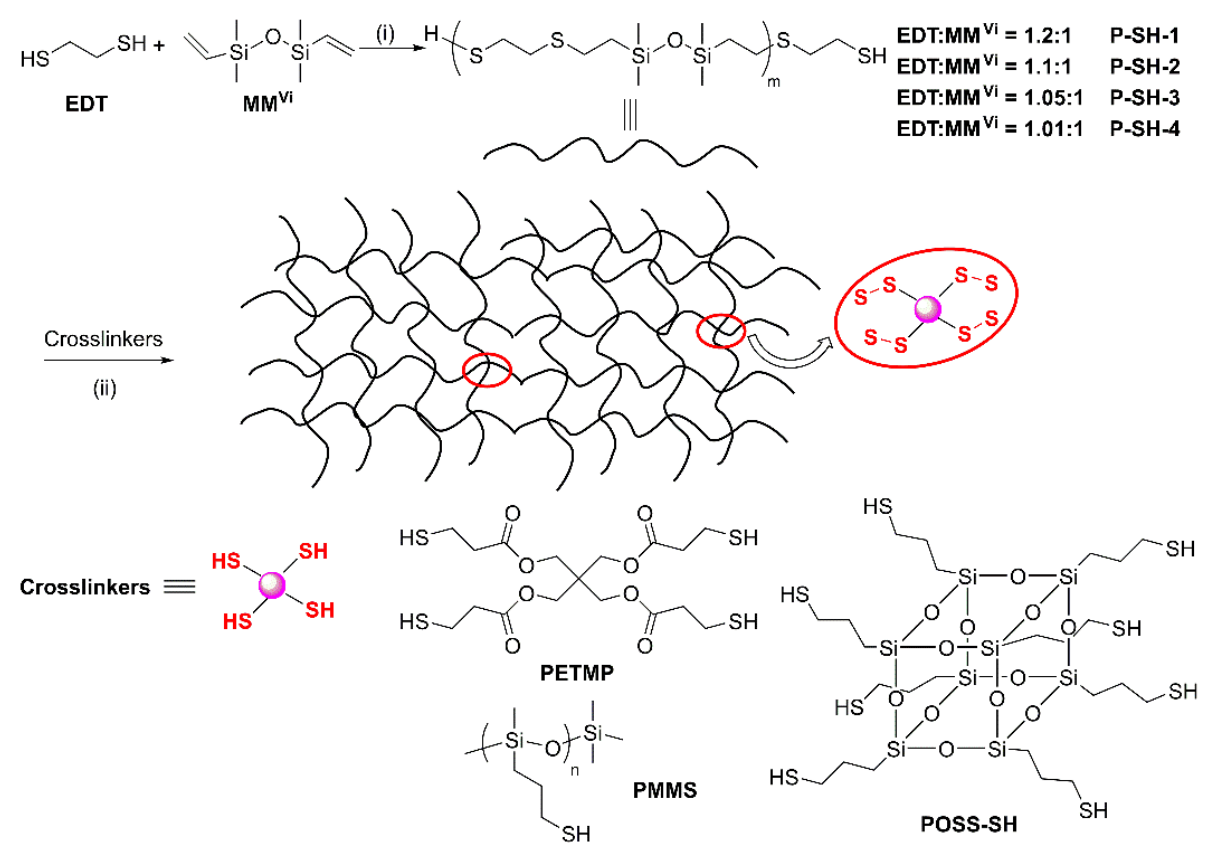

Scheme 1. Synthesis of disulfide-linked SEs. (i) DMPA, UV light, room temperature, 30 min; (ii) NaI, $30 \% \mathrm{H}_{2} \mathrm{O}_{2}$ aqueous solution, THF, room temperature.

The structure of SEs was determined by FT-IR spectroscopy (with SE-4 as an example). As shown in Figure 1, the peaks at 2963, 2913, 1064, 790, and $700 \mathrm{~cm}^{-1}$ in SE-4 can be attributed to $-\mathrm{CH},-\mathrm{Si}-\mathrm{O}-\mathrm{Si}-,-\mathrm{Si}-\mathrm{C}-$ and $-\mathrm{C}-\mathrm{S}-$ stretching vibrations. Compared to $\mathrm{P}-\mathrm{SH}-1$, the peak at ca. $2540 \mathrm{~cm}^{-1}$ assigned to $-\mathrm{SH}$ groups disappeared in the silicone elastomer, while a new and weak peak at ca. $472 \mathrm{~cm}^{-1}$, attributable to -S-S- stretching vibration, was observed. This finding indicates that the -SH groups were oxidized to -S-S- bonds, thus resulting in the formation of the crosslinking network. These results were consistent with our recent report [27]. 


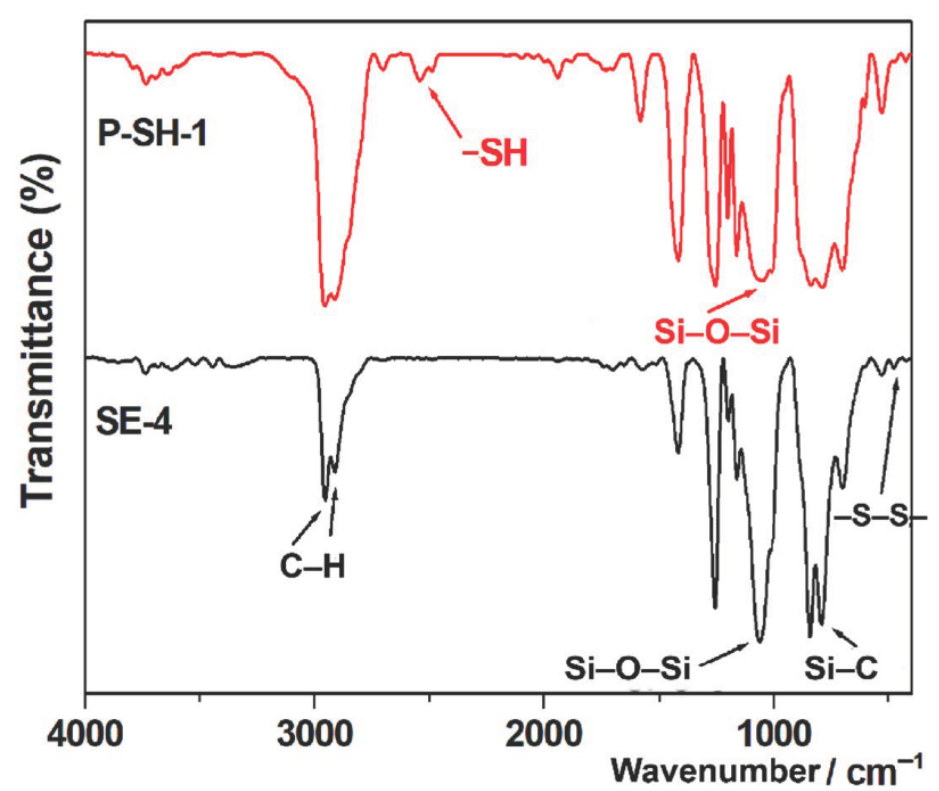

Figure 1. FT-IR of P-SH-1 and SE-4.

\subsection{Effects of Variables on the Formability and Mechanical Properties of SEs}

As mentioned above, the SEs were prepared by the thiol oxidation coupling reactions. To form a silicone elastomer with good quality, the crosslinking degree should be appropriate. If the degree is too low, the elastomers cannot be formed and the products appear as a viscous liquid. If the degree is too high, the products may be afforded a hard plastic with cracks. Thus, the effects of the amounts and species of crosslinkers on the formability and the mechanical properties of the resultant SEs were investigated.

Three crosslinkers, including PETMP, POSS-SH, and PMMS, were utilized to investigate the effects. As expected, the SEs cannot be afforded in the absence of crosslinker or with low amount of crosslinkers (e.g., SE-1, SE-2, SE-11, SE-15, and SE-19 in Table 1). For example, the products (SE- 1 and SE-2) were tacky when the amounts of PETMP were zero or $0.016 \mathrm{~g}$ and the other parameter included $2 \mathrm{~g}$ of P-SH-1, $8 \mathrm{~mL}$ of THF, $0.1 \mathrm{~mL}$ of $30 \%$ $\mathrm{H}_{2} \mathrm{O}_{2}$ in aqueous solution, and $0.05 \mathrm{~mL}$ of $\mathrm{NaI}$ in aqueous solutions $(0.1 \mathrm{~g} / \mathrm{mL})$. However, when the amount of PETMP is $0.1 \mathrm{~g}$, the elastomer can be afforded, but with many cracks (SE-5). Similar results were also found when using POSS-SH as the crosslinker (SE-6 to SE-9). However, when using PMMS, the elastomers cannot be afforded even if the amount of PMMS is $0.1 \mathrm{~g}$ (SE-10). This finding may be due to its high molecular weight. Unlike the small molecular crosslinkers PETMP and POSS-SH, PMMS with high molecular weight cannot effectively link the base polymer P-SHs, but might link themselves, thus leading to unsuccessful formation of the crosslinking network. In addition, when selecting P-SH-2 or P-SH-3 as the base polymers, the amount of crosslinkers on the formability of SEs were similar (SE-11 to SE-14 for P-SH-2, SE-15 to SE-21 for P-SH-3). Thus PETMP and POSS-SH were selected as the crosslinkers to investigate the effects. It is worthy to note that when using P-SH-4 as the base polymer, the SE cannot be formed even selecting the mixture of PETMP and POSS-SH as the crosslinker, and the product was afforded as a tacky liquid (SE-22). This finding can be explained by the low reactivity of -SH of P-SH-4 due to its high molecular weight and the thiol oxidation coupling reaction might mainly occur among the crosslinkers, not between the crosslinkers and P-SH-4.

The effect of crosslinker species on the mechanical properties was studied. SE-4 and SE-9 were selected because the contents of -SH groups based on PETMP and POSS-SH are nearly same. The results show that SE-4 with PETMP has a higher tensile strength of $0.17 \mathrm{MPa}$ and the elongation at break (218\%) than those (0.15 MPa and $85 \%)$ of SE-9 with POSS-SH (Figure 2). This finding is apparently due to the different structure of crosslinkers. POSS-SH is a rigid and cage molecule and has higher rigidity than PETMP. Thus, the 
formed crosslinking network is difficult to rotate. Moreover, POSS-SH can provide more crosslinking sites than PETMP. As a result, when using POSS-SH as a crosslinker, a rigid and concentrated crosslinking was formed in SE-4 and SE-4 exhibits a better mechanical property than SE-9.

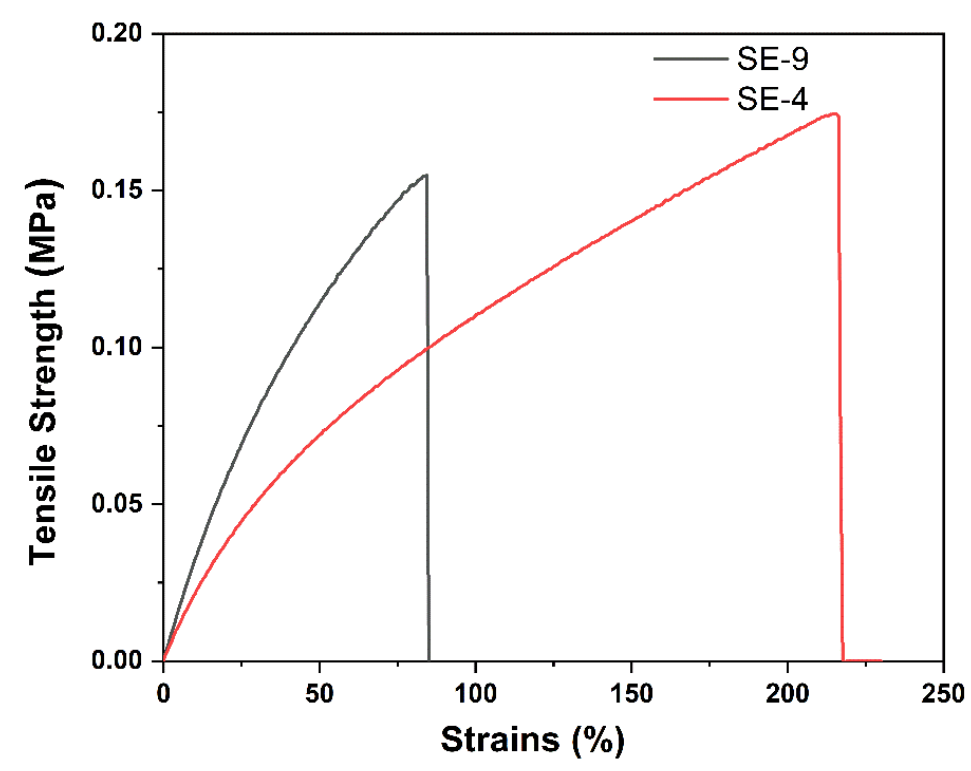

Figure 2. Tensile curves of SE-4 and SE-9.

The effect of the amount of crosslinkers on the mechanical properties was further studied and the elastomers with POSS-SH as the crosslinker were selected as examples (Figure 3). With an increment of the amount of POSS-SH, the elongation at break gradually decreased from $545 \%$ (SE-6) to $85 \%$ (SE-9), while the trend change of the tensile strength was not regular and SE-8 with the moderate amount of crosslinker exhibited the highest tensile strength of $0.23 \mathrm{MPa}$. This finding can be explained by the tuned crosslinking density induced by the varied amount of crosslinker. It is known that when increasing the amount of crosslinker, the crosslinking sites would increase, leading to enhanced tensile strength and elongation at break. However, if the amount is too high, the excessive crosslinking will lead to cracks in the elastomer and make the tensile strength decrease.

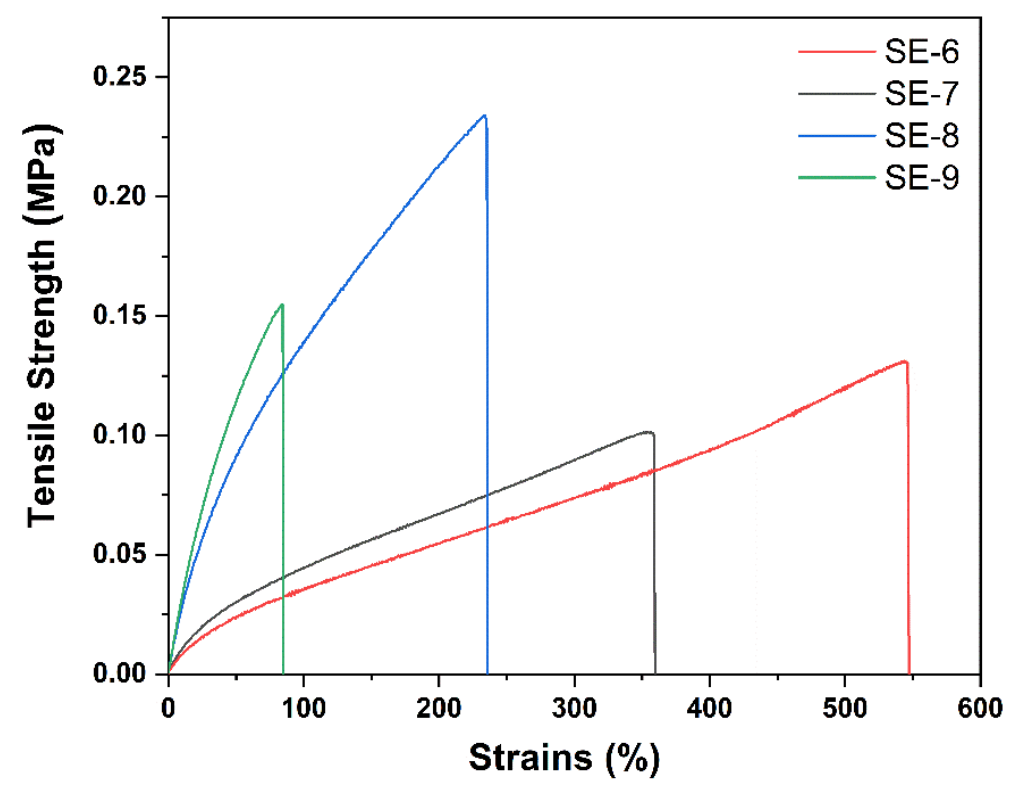

Figure 3. The effect of the cross-linker content on stress of the elastomer. 


\subsection{Thermal Stability}

The thermal stability of these elastomers was evaluated by thermogravimetric analysis (TGA) and differential scanning calorimetry (DSC) because it plays an important role in their applications. The TGA experiments were conducted at a heating rate of $10 \mathrm{~K} / \mathrm{min}$ under $\mathrm{N}_{2}$. Figure 4 shows the TGA curves of SE-2, SE-4, SE-6, SE-12, and SE-18 as examples. It was found that these elastomers exhibit high thermal stability with the $\mathrm{T}_{\mathrm{d}, 5 \%}(5 \%$ weight loss temperature) at ca. $300{ }^{\circ} \mathrm{C}$. After that, the elastomers quickly decompose and fully decompose at ca. $400{ }^{\circ} \mathrm{C}$. This finding may be due to the decomposition of disulfide bonds, which induced the decomposition of sulfur-containing heterochain polysiloxane and the formation of some cyclic monomers.

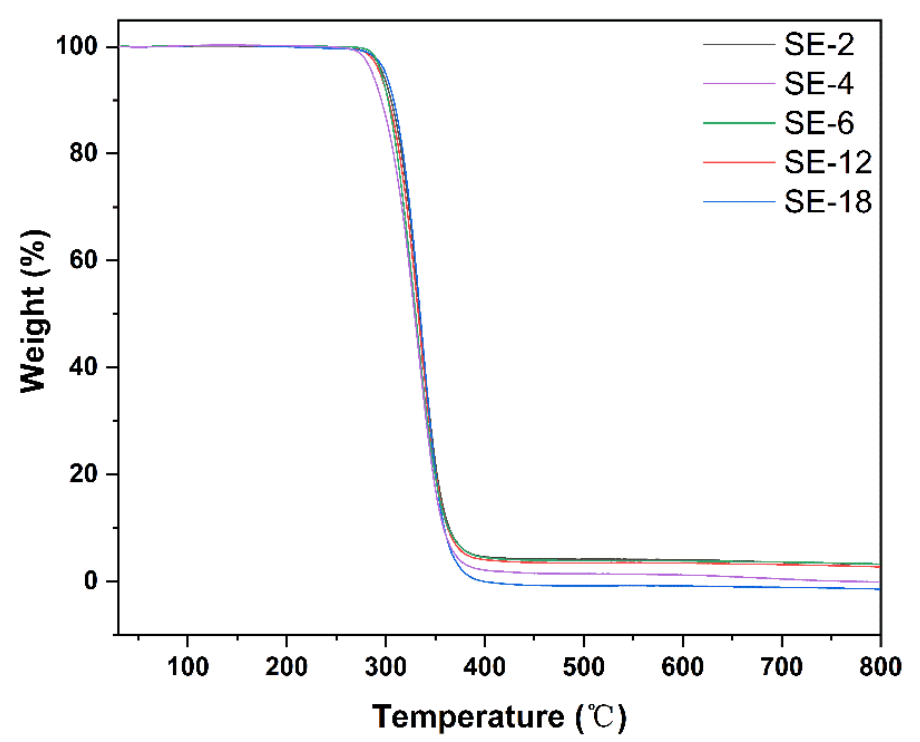

Figure 4. TGA curves of SE-2, SE-4, SE-6, SE-12 and SF-18 at a heating rate of $10^{\circ} \mathrm{C} \mathrm{min}^{-1}$ in nitrogen.

Figure 5 shows the DSC curves of SE-3, SE-12, and SE-17 as examples. The glass transition temperatures of these elastomers are at ca. $-60^{\circ} \mathrm{C}$, indicating their low temperature resistance and application in low temperature circumstances. In addition, SE-3 and SE-17 exhibit a crystalline peak at 39 and $20^{\circ} \mathrm{C}$, respectively. The finding is apparently due to the heterochain polysiloxane, which is different from the conventional pure polysiloxane chain [4].

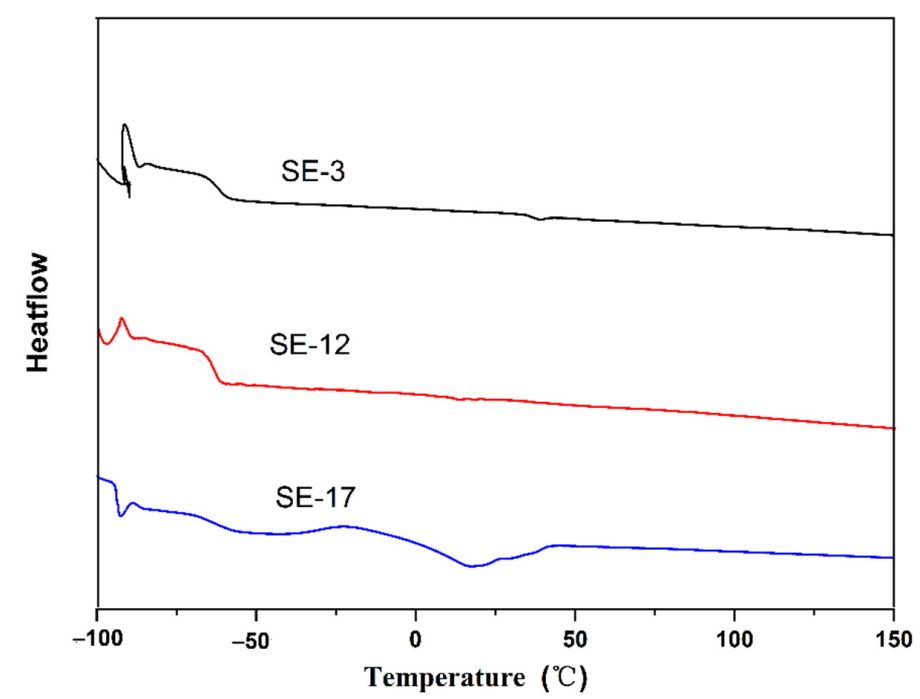

Figure 5. The DSC curves of SE-3, SE-12, and SE-17. 


\subsection{Self-Healing Properties of SES}

Due to the presence of reversible disulfide bonds in the networks, these SEs can be expected to possess self-healing properties. Thus, the self-healing property was evaluated using SE-3 as an example. UV light and heating were applied as the healing conditions. Firstly, the elastomer was cut into pieces with a knife. The resultant pieces were placed between two glass sheets and the sheets were clamped. After exposing the pieces to UV light (the wavelength was $365 \mathrm{~nm}$ ) for $30 \mathrm{~min}$ or heating them at $150{ }^{\circ} \mathrm{C}$ for $2 \mathrm{~h}$, the elastomers re-formed (Figure 6). These results indicate that the elastomer can be healed.

(a)
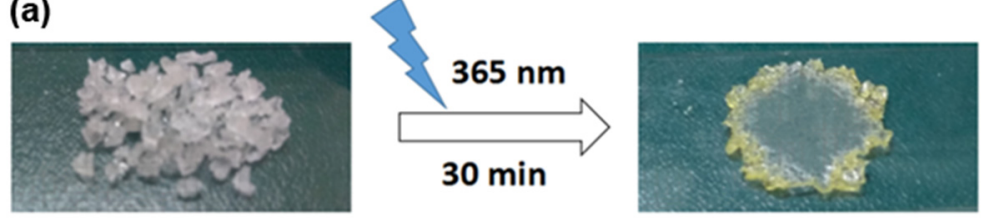

(b)
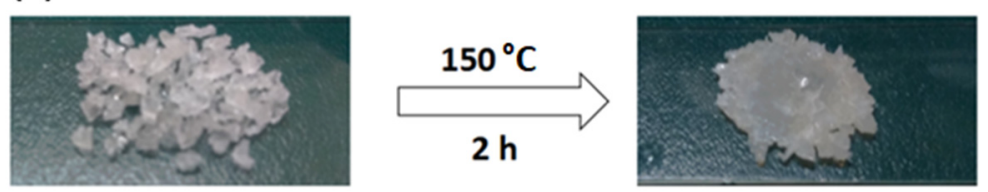

Figure 6. Self-repairing experiments of SE-3 under UV light (365 nm) for $30 \mathrm{~min}(\mathbf{a})$ or heating at $150{ }^{\circ} \mathrm{C}$ for $2 \mathrm{~h}(\mathbf{b})$.

The healing efficiency was further evaluated using SE-3 as an example. It was found that if there was no pressure force during the self-healing process under UV light, the elastomer could not be effectively repaired. However, under heating conditions at $150{ }^{\circ} \mathrm{C}$ for $2 \mathrm{~h}$, the elastomer can be effectively healed. As shown in Figure 7, the tensile strength is $0.11 \mathrm{MPa}$, which is lower than the original value of $0.15 \mathrm{MPa}$. This result means the self-healing efficiency is $>70 \%$, thus indicating that the present elastomer can be effectively healed after the destruction.

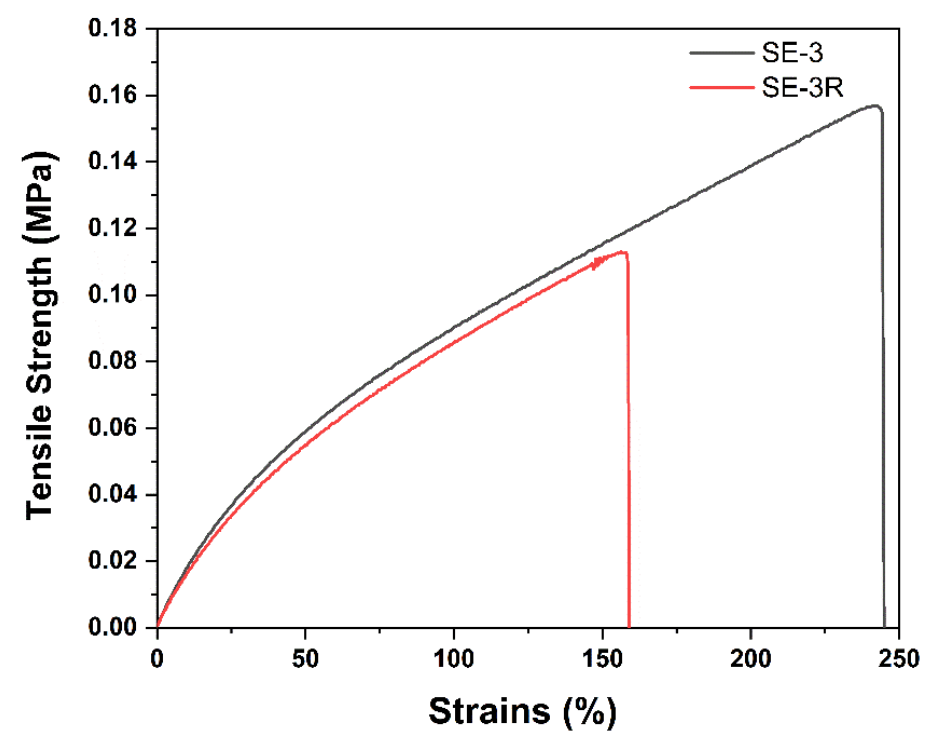

Figure 7. Stress-strain curves before and after self-healing of SE-3. SE-3R represents the elastomer after repair.

\subsection{Application as Adhesives}

These elastomers were applied as adhesives and SE- 4 was selected as an example. The experiment was conducted by placing a $6 \mathrm{~mm} \times 6 \mathrm{~mm}$ elastomer between two overlapping $25 \mathrm{~mm} \times 75 \mathrm{~mm}$ glass sheets. The resultant sheets were clamped using two clamps and 
exposed to UV light for 30 min while maintaining the contact pressure. After removing the UV irradiation, the adhesive glass sheets can hold a maximum $200 \mathrm{~g}$ of weight, while the glass sheets will break under higher weight. Then the glass sheets were re-exposed to UV light, and the weight dropped because of the decreased adhesive strength (Figure 8). This finding is apparently due to the reversible crosslinking network in the presence of dynamic disulfide bonds. It is delightful that the adhesive and detached process can be repeated many times. These results suggest that these elastomers can be promisingly applied as reversible adhesives.
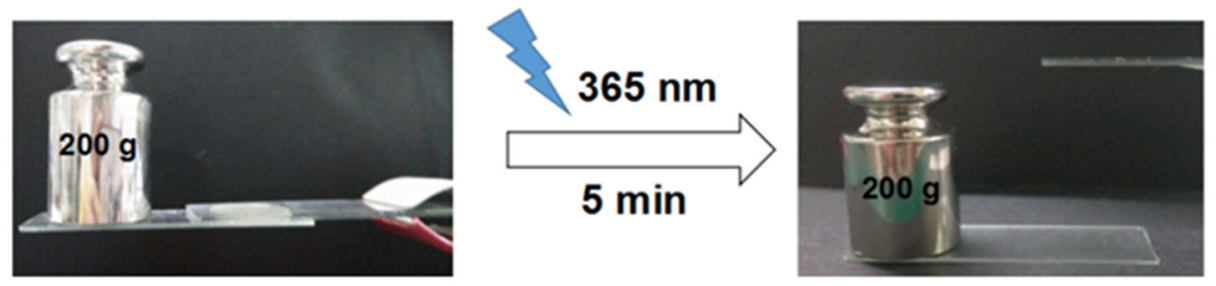

Figure 8. The bonding performance test.

\section{Conclusions}

Novel self-healing disulfide-linked silicone elastomers have been successfully created by introducing a thiol oxidation coupling reaction as a crosslinking strategy when using thiol-terminated sulfur-containing heterochain polysiloxanes (P-SHs) as the base polymers and three thiol-containing compounds or polymers, including PETMP, POSS-SH, and PMMS, as the crosslinkers. The crosslinking process is mild and rapid. The effects of the crosslinker species and amounts on the formability and mechanical properties were investigated. The SEs can be readily repaired by heating at $150{ }^{\circ} \mathrm{C}$ for $2 \mathrm{~h}$ or exposure to UV radiation for $30 \mathrm{~min}$ with a healing efficiency of $>70 \%$, indicating their self-healing property. For application, they can be used as reversible adhesives for bonding glass sheets, which can hold $200 \mathrm{~g}$ of weight. To the best of our knowledge, this work represents the first example of a thiol oxidation coupling reaction as a crosslinking strategy for the construction of self-healing silicone elastomers. This simple and effective strategy can certainly be expanded to construct other self-healing elastomers based on thiol-containing base polymers and crosslinkers and explore the applications of these novel materials.

Author Contributions: Conceptualization, D.W. and C.Z.; methodology, Y.H., J.Y., D.W. and C.Z.; formal analysis, Y.H. and J.Y.; investigation, Y.H., J.Y., S.F., D.W. and C.Z.; data curation, Y.H. and J.Y.; writing —original draft preparation, Y.H. and J.Y.; writing—review and editing, D.W., S.F. and C.Z.; visualization, Y.H. and J.Y.; supervision, D.W. and C.Z.; project administration, C.Z.; funding acquisition, D.W. and C.Z. All authors have read and agreed to the published version of the manuscript.

Funding: This research was funded by the National Natural Science Foundation of China (U2030203), the Fluorine Silicone Materials Collaborative Fund of Shandong Provincial Natural Science Foundation (ZR2020LFG011), the Shandong Provincial Natural Science Foundation (ZR2019MB028), and the Young Scholars Program of Shandong University.

Institutional Review Board Statement: Not applicable.

Informed Consent Statement: Not applicable.

Data Availability Statement: Not applicable.

Conflicts of Interest: The authors declare no conflict of interest.

\section{References}

1. Yilgör, E.; Yilgör, I. Silicone containing copolymers: Synthesis, properties and applications. Prog. Polym. Sci. 2014, 39, 1165-1195. [CrossRef]

2. Eduok, U.; Faye, O.; Szpunar, J. Recent developments and applications of protective silicone coatings: A review of PDMS functional materials. Prog. Org. Coat. 2017, 111, 124-163. [CrossRef] 
3. Liu, J.; Yao, Y.; Li, X.; Zhang, Z. Fabrication of advanced polydimethylsiloxane-based functional materials: Bulk modifications and surface functionalizations. Chem. Eng. J. 2020, 408, 127262. [CrossRef]

4. Wolf, M.P.; Salieb-Beugelaar, G.B.; Hunziker, P. PDMS with designer functionalities-Properties, modifications strategies, and applications. Prog. Polym. Sci. 2018, 83, 97-134. [CrossRef]

5. Qi, D.; Zhang, K.; Tian, G.; Jiang, B.; Huang, Y. Stretchable Electronics Based on PDMS Substrates. Adv. Mater. 2021, $33,2003155$. [CrossRef] [PubMed]

6. Yi, B.; Wang, S.; Hou, C.; Huang, X.; Cui, J.; Yao, X. Dynamic siloxane materials: From molecular engineering to emerging applications. Chem. Eng. J. 2021, 405, 127023. [CrossRef]

7. Rus, D.; Tolley, M.T. Design, fabrication and control of soft robots. Nature 2015, 521, 467-475. [CrossRef]

8. Lv, C.; Zhao, K.; Zheng, J. A Highly Stretchable Self-Healing Poly(dimethylsiloxane) Elastomer with Reprocessability and Degradability. Macromol. Rapid Commun. 2018, 39, 201700686. [CrossRef] [PubMed]

9. Lai, J.-C.; Mei, J.-F.; Jia, X.-Y.; Li, C.-H.; You, X.-Z.; Bao, Z. A Stiff and Healable Polymer Based on Dynamic-Covalent Boroxine Bonds. Adv. Mater. 2016, 28, 8277-8282. [CrossRef]

10. Kathan, M.; Kovaricek, P.; Jurissek, C.; Senf, A.; Dallmann, A.; Thuenemann, A.F.; Hecht, S. Control of Imine Exchange Kinetics with Photoswitches to Modulate Self-Healing in Polysiloxane Networks by Light Illumination. Ang. Chem. Inter. Ed. 2016, 55, 13882-13886. [CrossRef] [PubMed]

11. Nasresfahani, A.; Zelisko, P.M. Synthesis of a self-healing siloxane-based elastomer cross-linked via a furan-modified polyhedral oligomeric silsesquioxane: Investigation of a thermally reversible silicon-based cross-link. Polym. Chem. 2017, 8, $2942-2952$. [CrossRef]

12. Zhang, H.; Cai, C.; Liu, W.; Li, D.; Zhang, J.; Zhao, N.; Xu, J. Recyclable Polydimethylsiloxane Network Crosslinked by Dynamic Transesterification Reaction. Sci. Rep. 2017, 7, 11833. [CrossRef] [PubMed]

13. Li, C.-H.; Wang, C.; Keplinger, C.; Zuo, J.-L.; Jin, L.; Sun, Y.; Zheng, P.; Cao, Y.; Lissel, F.; Linder, C.; et al. A highly stretchable autonomous self-healing elastomer. Nat. Chem. 2016, 8, 618. [CrossRef] [PubMed]

14. Zhang, K.; Sun, J.; Song, J.; Gao, C.; Wang, Z.; Song, C.; Wu, Y.; Liu, Y. Self-Healing $\mathrm{Ti}_{3} \mathrm{C}_{2}$ MXene/PDMS Supramolecular Elastomers Based on Small Biomolecules Modification for Wearable Sensors. ACS Appl. Mater. Interf. 2020, 12, 45306-45314. [CrossRef] [PubMed]

15. Fawcett, A.S.; Brook, M.A. Thermoplastic Silicone Elastomers through Self-Association of Pendant Coumarin Groups. Macromolecules 2014, 47, 1656-1663. [CrossRef]

16. Rambarran, T.; Bertrand, A.; Gonzaga, F.; Boisson, F.; Bernard, J.; Fleury, E.; Ganachaud, F.; Brook, M.A. Sweet supramolecular elastomers from [small alpha], [small omega]-([small beta]-cyclodextrin terminated) PDMS. Chem. Commun. 2016, 52, 6681-6684. [CrossRef]

17. Griebel, J.J.; Nguyen, N.A.; Namnabat, S.; Anderson, L.E.; Glass, R.S.; Norwood, R.A.; Mackay, M.E.; Char, K.; Pyun, J. Dynamic Covalent Polymers via Inverse Vulcanization of Elemental Sulfur for Healable Infrared Optical Materials. ACS Macro Lett. 2015, 4, 862-866. [CrossRef]

18. Michal, B.T.; Spencer, E.J.; Rowan, S.J. Stimuli-Responsive Reversible Two-Level Adhesion from a Structurally Dynamic ShapeMemory Polymer. ACS Appl. Mater. Interf. 2016, 8, 11041-11049. [CrossRef]

19. Michal, B.T.; Jaye, C.A.; Spencer, E.J.; Rowan, S.J. Inherently Photohealable and Thermal Shape-Memory Polydisulfide Networks. ACS Macro Lett. 2013, 2, 694-699. [CrossRef]

20. Xu, W.M.; Rong, M.Z.; Zhang, M.Q. Sunlight driven self-healing, reshaping and recycling of a robust, transparent and yellowingresistant polymer. J. Mater. Chem. A 2016, 4, 10683-10690. [CrossRef]

21. Amamoto, Y.; Otsuka, H.; Takahara, A.; Matyjaszewski, K. Self-Healing of Covalently Cross-Linked Polymers by Reshuffling Thiuram Disulfide Moieties in Air under Visible Light. Adv. Mater. 2012, 24, 3975-3980. [CrossRef] [PubMed]

22. Lei, Z.Q.; Xiang, H.P.; Yuan, Y.J.; Rong, M.Z.; Zhang, M.Q. Room-Temperature Self-Healable and Remoldable Cross-linked Polymer Based on the Dynamic Exchange of Disulfide Bonds. Chem. Mater. 2014, 26, 2038-2046. [CrossRef]

23. Xiang, H.P.; Rong, M.Z.; Zhang, M.Q. A facile method for imparting sunlight driven catalyst-free self-healability and recyclability to commercial silicone elastomer. Polymer 2017, 108, 339-347. [CrossRef]

24. Liu, Y.; Yuan, J.; Zhang, K.; Guo, K.; Yuan, L.; Wu, Y.; Gao, C. A novel type of self-healing silicone elastomers with reversible cross-linked network based on the disulfide, hydrogen and metal-ligand bonds. Prog. Org. Coat. 2020, 144, 105661. [CrossRef]

25. Shan, S.; Lin, Y.; Zhang, A. Stretchable, robust and reprocessable poly(siloxane-urethanes) elastomers based on exchangeable aromatic disulfides. Polymer 2021, 221, 123588. [CrossRef]

26. Zhao, L.; Yin, Y.; Jiang, B.; Guo, Z.; Qu, C.; Huang, Y. Fast room-temperature self-healing siloxane elastomer for healable stretchable electronics. J. Colloid Interf. Sci. 2020, 573, 105-114. [CrossRef]

27. Yan, J.; Cao, J.; Xue, L.; Feng, S.; Zhang, H.; Wang, D. Thiol Oxidative Coupling Synthesis of Silicone Foams for Oil/Water Separation. ACS Appl. Polym. Mater. 2020, 2, 1634-1643. [CrossRef]

28. Li, L.; Liu, H. Rapid Preparation of Silsesquioxane-Based Ionic Liquids. Chem. Eur. J. 2016, 22, 4713-4716. [CrossRef] 